\title{
Robust Real-time Self-Mixing Interferometric Laser Vibration Sensor with Embedded MEMS Accelerometer
}

\author{
Usman Zabit ${ }^{\mathrm{a}-\mathrm{b}}$, Olivier D. Bernal ${ }^{\mathrm{a}-\mathrm{b}}$, and Thierry Bosch ${ }^{\mathrm{a}-\mathrm{b}}$ \\ ${ }^{a}$ CNRS, LAAS, 7 avenue du colonel Roche, F-31400 Toulouse, France \\ ${ }^{b}$ Univ de Toulouse, INP, LAAS, F-31400 Toulouse, France
}

\begin{abstract}
In this paper, we present a real-time implementation of a Self-Mixing (SM) interferometric laser diode (LD) based vibration sensor coupled with an embedded MEMS (microelectromechanical system) accelerometer. Such a sensor allows measuring correct target movements even when the LD based SM sensor is subject to extraneous movements. This results in a vibration sensing system that can be used for embedded applications as there is no more need of keeping the sensor stationary. Such an approach opens the way for the use of such laser sensors in conditions where the use of anti-vibration support is not available or possible. The proposed data fusion between a MEMS accelerometer and a LD based SM sensor results in a robust, compact and low-cost sensing system.
\end{abstract}

Keywords: embedded sensing, vibration measurement, displacement measurement, selfmixing interferometry, accelerometer, laser diode.

\section{INTRODUCTION}

Self-mixing (SM) or optical feedback interferometry technique has been investigated during the last two decades [1-3] for distance [4], velocity [5], vibration [6] and displacement [7] sensing applications as it results in a simple, compact, self-aligned, and low-cost sensor that becomes attractive not only for industrial use but also for large-scale consumer applications [5].

SM effect occurs in a laser when a part of the beam backscattered by a target is coupled back into the laser cavity and causes interference with the emitted beam, thus modifying the spectral properties of the laser. The variations in the optical output power of the laser diode $P(t)$ caused by this optical feedback can be written as [8]:

$$
P(t)=P_{0}\left[1+m \cdot \cos \left(x_{F}(t)\right)\right]
$$

where $P_{0}$ is the emitted optical power under free-running conditions, $m$ is the modulation index and $x_{F}(t)$ is the laser output phase in the presence of feedback, given by:

$$
x_{F}(t)=2 \pi \frac{D(t)}{\lambda_{F}(t) / 2}
$$


where $D(t)$ is the target displacement.

The emission wavelength subject to feedback $\lambda_{F}(t)$ is given by the phase equation [8]:

$$
x_{0}(t)-x_{F}(t)-C \sin \left[x_{F}(t)+\arctan (\alpha)\right]=0
$$

where $\alpha$ is the linewidth enhancement factor and $x_{0}(t)$ is the laser output phase in the absence of feedback, given by:

$$
x_{0}(t)=2 \pi \frac{D(t)}{\lambda_{0} / 2}
$$

where $\lambda_{0}$ is the emission wavelength under free running conditions. The feedback coupling factor $\mathrm{C}$ is given by [3]:

$$
C=\frac{\tau_{D}}{\tau_{L}} \gamma \sqrt{1+\alpha^{2}} \kappa_{e x t}
$$

where $\tau_{L}$ and $\tau_{D}$ are the round trip times in the internal and external cavities respectively, $\gamma$ is the coupling efficiency and $\kappa_{e x t}$ depends linearly on the surface reflectivity of the target.

The C parameter plays an important role in the SM interferometry as variations in C cause changes in the so called SM operating regimes varying from weak [9] to moderate [10] to strong feedback [11], where each regime would require specific signal processing for the sake of displacement retrieval [12]. For the present work, care has been taken to maintain the SM signal in the moderate regime with $\mathrm{C}$ ranging from 1 to 2 as the resulting SM signal requires simplified processing and is not affected by the fringe-loss issue [13].

It needs to be mentioned that SM displacement sensors have traditionally needed a stationary support (such as an optical table and/or anti-vibration material) to guarantee an accurate target measurement as any movement of the SM sensing laser system can corrupt the true target displacement measurement. Such a situation has thus restricted the use of SM sensors for embedded applications. (Note that this argument is valid for all sensors in general.)

So, in order to overcome such a limitation, a Solid-State Accelerometer (SSA) coupled SelfMixing (SSA-SM) sensing system has been recently proposed [14] that measures true target displacement even if the laser sensor is itself subject to a displacement (noted as $D_{S}$ ). A schematic diagram of such a SSA-SM sensing system is presented in Fig. 1. The solid-state MEMS (micro-electro-mechanical system) accelerometer measures the laser sensor head displacement and a subsequent subtraction of this displacement from the global (falsified) SM displacement provides the true target displacement. 


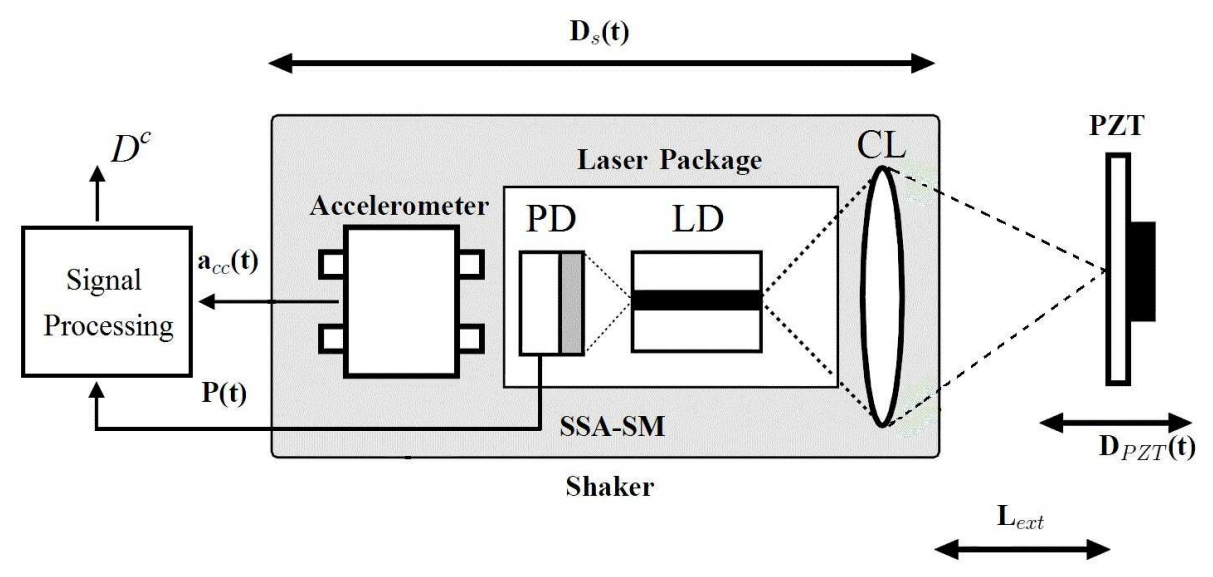

FIGURE 1. Schematic block diagram of the Solid-State Accelerometer (SSA) coupled SelfMixing (SSA-SM) sensing system: photodiode (PD), laser diode (LD), collimating lens (CL), and piezoelectric transducer (PZT).

In this paper, after a presentation of the signal processing needed for a SSA-SM sensing system, we analyze the impact of the employed signal processing as well as the key accelerometer characteristics on the overall SSA-SM system performance in terms of measurement precision and bandwidth. Then, the paper would detail the design of a real-time SSA-SM sensing system. The presented real-time SM vibration sensor system is based on a LD package from Sanyo (DL7140) emitting at $\lambda=785 \mathrm{~nm}$ with an output power of $60 \mathrm{~mW}$. The accelerometer LIS344ALH is from ST ${ }^{\circledR}$ with a typical noise resolution of $50 \mu \mathrm{g} / \sqrt{\mathrm{Hz}}$ and a fullscale range of $\pm 2 \mathrm{~g}$. Finally, the results based on this real-time sensing prototype would be presented and evaluated in order to highlight the sources of error still resident in the final output of the sensor.

Let us now start with a presentation of the signal processing used for the real-time SSA-SM sensing system.

\section{SIGNAL PROCESSING}

Figure 2 details the steps undertaken for the correct measurement of true target displacement even in presence of other displacements affecting the SM sensor. These consist of three main parts as explained below. 


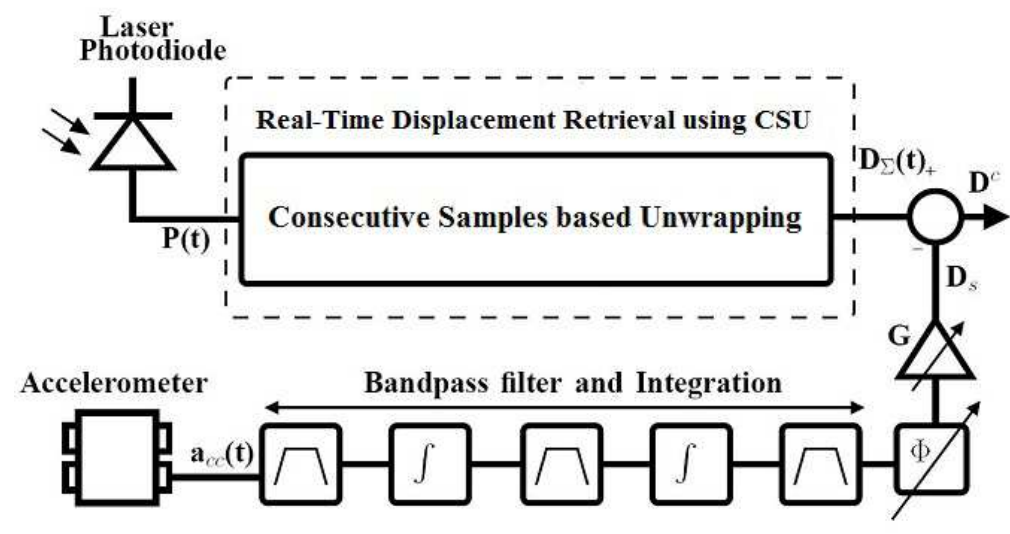

FIGURE 2. Schematic block diagram of the robust real-time Self-Mixing interferometric laser vibration sensor with embedded MEMS accelerometer.

\section{Self-Mixing Signal based Real-Time Displacement Retrieval}

The variations in the optical output power of the laser diode $\mathrm{P}(\mathrm{t})$ are monitored through the built-in photodiode (PD) contained in the laser diode (LD) package. For real-time SSA-SM sensing system, SM displacement retrieval was done by using the Consecutive Samples based Unwrapping (CSU) method [15]. Note that in this paper, this displacement retrieved from the signal $\mathrm{P}(\mathrm{t})$ has been denoted as $\mathrm{D}_{\Sigma}$ since it is the sum of the true target displacement $\mathrm{D}_{\mathrm{PZT}}$ and the displacement undergone by the sensor $\mathrm{D}_{\mathrm{s}}$.

The working of CSU method is shown in Fig. 3. It starts by retrieving the SM based displacement through fringe-counting resulting in an initial precision of $\lambda / 2$. This initial step is quite similar to the initial step of the PUM [10] or the fully analog SM processing method [16]. Then, instead of doing an iterative joint-estimation as in PUM, it uses the local SM signal unwrapping (by adding the fringe-counting based staircase signal to the normalized SM signal) as in [16]. Thus, it is able to retrieve SM based displacement with a precision better than $\lambda / 2$.

In order to process the SM signal in real-time, the fully analog SM processing technique may have been considered. However, keeping in mind the non-harmonic nature of $\mathrm{D}_{\Sigma}$, it is important to use an approach capable of processing non-harmonic movements without suffering from drift effects. So, for the prototype, the SM signal has been processed in real-time using the CSU method [15].

As compared to a complex approach such as the PUM that is based on joint-estimation of SM parameters requiring iterative optimization routines, the consecutive-sampled unwrapping has the great benefit of requiring only two SM samples to update the corresponding displacement output. This lack of parameter estimation allows a fast real-time displacement measurement. It is thus seen that the ensuing results, though not as precise as the final output of PUM, still compare favorably with the PUM displacement [15].

For the prototype, an integrated micro-converter AduC7020 from Analog Devices ${ }^{\circledR}$ working at 40 MIPS has been used that has integrated Analog-to-Digital (A/D) and Digital-to-Analog $(\mathrm{D} / \mathrm{A})$ converters. The incoming signal $\mathrm{P}(\mathrm{t})$ is processed into the output displacement signal $\mathrm{D}(\mathrm{t})$ at $125 \mathrm{KHz}$ with an approximately $\lambda / 8$ precision. So, the output displacement is updated every $8 \mu \mathrm{s}$. 
As far as the maximum measurable target speed using this prototype is concerned, the experimental data has showed that 20 samples per SM fringe are normally needed in order to correctly detect the fringe-discontinuity of each SM fringe having its associated noise. This then provides the maximum measurable target speed of $2.45 \mathrm{~mm} / \mathrm{s}$ for the real-time displacement sensor [15].

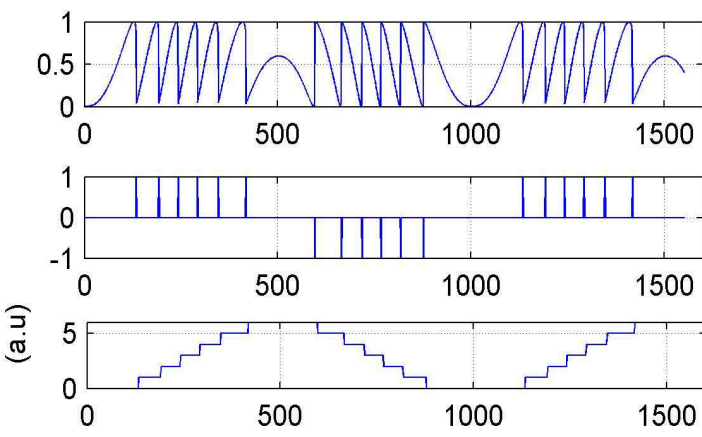

(a)

(b)

(c)



(d)

FIGURE 3. Displacement retrieval through Consecutive-Samples based Unwrapping [15]. (a) normalized SM signal corresponding to $\mathrm{C}=1.5$, (b) fringe discontinuity detection, (c) staircase signal representing the fringe count and (d) the retrieved signal proportional to target excitation signal obtained by the addition of signals (a) and (c).

\section{Displacement Extraction from the Acceleration Signal}

The displacement undergone by the sensor itself and measured through the acceleration signal $\left(D_{s}\right)$ is obtained by using the SSA. In order to do so, the acceleration signal $a_{c c}(t)$ is filtered and integrated twice, as indicated in the lower half of Fig. 2. The band-pass filtering is done over the operating bandwidth of $20 \mathrm{~Hz}-500 \mathrm{~Hz}$ so that low-frequency drifts that can falsify the subsequent integration steps as well as high frequency acceleration signal saturation can be effectively avoided.

The choice of a given low cut-off frequency is closely related to the overall SSA-SM sensing system measurement precision. As displacement extraction from acceleration signal involves double integration, so the noise power spectrum density of the displacement $S_{D}$ can be given in the frequency domain as [14]

$$
S_{D}(s)=\frac{S_{a c c}(s)}{s^{4}}
$$

Consequently, the displacement resolution of the SSA signal depends on the low cutoff frequency of the system. For a $20 \mathrm{~Hz}$ lower cutoff, LIS344ALH is thus expected to introduce an RMS error of approximately 40nm [17]. 
For the real-time prototype, the integration and filtering stages (indicated in Fig. 2) needed for extracting displacement from LIS344ALH have been based on standard analog OP-AMP based circuits.

\section{Phase and Gain Matching}

In order to match the two signals $\left(D_{\Sigma}\right.$ and $\left.D_{s}\right)$, phase $(\phi)$ and gain $(G)$ corrections need to be done. This is necessary as both signals i.e. the self-mixing signal and the acceleration signal pass through their respective signal amplification, acquisition, and processing stages all of which may introduce differences in phase and gain between the two signals.

Finally, the signal matching of the two signals $\left(D_{\Sigma}\right.$ and $\left.D_{s}\right)$ is followed by the last step of their subtraction that allows us to obtain the corrected displacement $\mathrm{D}^{\mathrm{c}}$.

For the real-time prototype, the gain stages and phase correcting filters have also been developed using the standard analog OP-AMP based circuits.

\section{PROTOTYPE ERROR ANALYSIS}

As there are three main signal processing steps for the presented SSA-SM system so each of these three can introduce error to the complete system thus reducing the final precision of the SSA-SM system. Let us look into these one by one.

Firstly, the method used to retrieve displacement information from a given SM signal would contribute an error to the final SSA-SM system precision. The use of CSU method for retrieving displacement from a SM sensor signal based on a $785 \mathrm{~nm}$ wavelength LD would thus introduce an error of approximately $80 \mathrm{~nm}$. This error value may further increase in the case of a nonperfect normalization of the SM signal.

Secondly, the noise density of the used SSA (LIS344ALH in the present case having a noise density of $50 \mu \mathrm{g} / \mathrm{VHz}$ as reported in datasheets) has a direct impact on the displacement measurement resolution for a given low cutoff frequency of the system bandwidth [16]. It can then be estimated that the displacement resolution for LIS344ALH will be of approximately $80 \mathrm{~nm}$ for a $20 \mathrm{~Hz}$ lower cutoff.

Thirdly, we need to consider the possible influence of a mismatch in phase and gain coefficients. As a perfect elimination of an extraneous movement acting on SSA-SM system supposes a perfect phase and gain matching so it is easy to see that any mismatch of phase and gain coefficients would result in a residue after a subtraction of $D \Sigma$ and Ds. Such a residual signal would then directly contribute in reducing the final SSA-SM sensing system precision. Denoting gain mismatch as $\Delta \mathrm{G}$ and phase mismatch as $\Delta \phi$ and assuming a sinusoidal extraneous motion with $10 \mu \mathrm{m}$ amplitude, it can then be calculated that a residual sinusoidal signal of $100.38 \mathrm{~nm}$ amplitude would appear in the final output of SSA-SM system for $\Delta \mathrm{G}=0.5 \%$ and $\Delta \phi=0.5$ degrees. Figure 4 presents the simulation results of the peak amplitude of such a residue as a percent of the extraneous sinusoidal motion amplitude acting on the SSA-SM system for varying $\Delta \mathrm{G}$ and $\Delta \phi$. It can thus be seen that an increased mismatch would have a drastic effect on the overall system precision even if SM displacement retrieval and acceleration signal processing were perfect. 


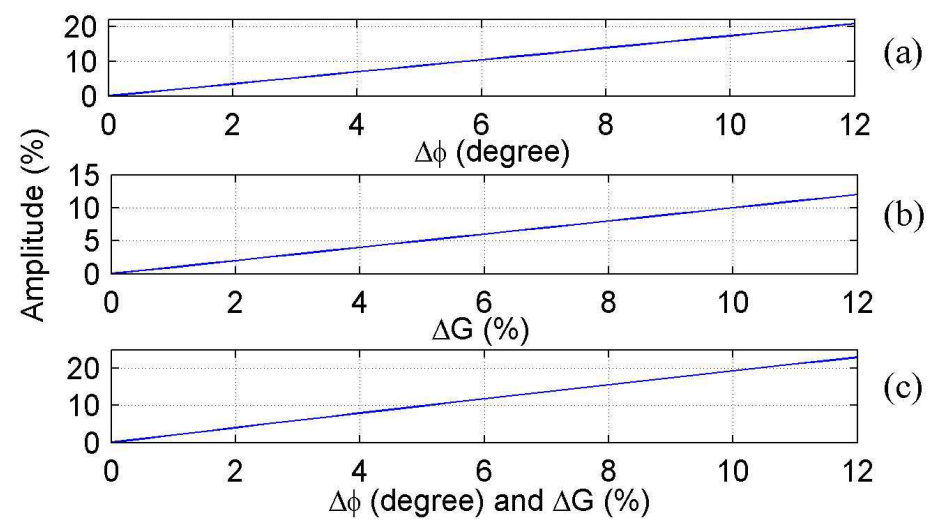

FIGURE 4. Influence of a mismatch in Gain and Phase coefficients on the elimination of extraneous movement acting on SSA-SM sensing system. (a) Percent amplitude of [ $\sin (\omega t)$ $\sin (\omega t-\Delta \phi)]$, (b) percent amplitude of [ $\sin (\omega t)-(1-\Delta \mathrm{G}) \sin (\omega \mathrm{t})]$, and (c) percent amplitude of $[\sin (\omega \mathrm{t})-(1-\Delta \mathrm{G}) \sin (\omega \mathrm{t}-\Delta \phi)]$.

Thus, it can be said that the imprecision $\xi^{c}$ in the final SSA-SM system output $D^{c}$ would have uncorrelated contributions from the above-mentioned three errors and can be expressed as

$$
\xi^{c} \approx \sqrt{\xi_{S M}^{2}+\xi_{a c c}^{2}+\xi_{\Delta}^{2}}
$$

where $\xi_{\mathrm{SM}}$ is the error in SM displacement extraction, $\xi_{\mathrm{acc}}$ is the error brought by a given accelerometer, and $\xi_{\Delta}$ is the gain and phase mismatch error respectively. Note that $\xi^{c}$ may also be affected by stray mechanical, data acquisition, and electronic noise though these effects have not been quantified in the present study.

\section{EXPERIMENTAL RESULTS}

A photograph of the real-time SSA-SM sensing system prototype is shown in Fig. 5. The prototype casing has been opened to show different components used in it. The component cost of the complete prototype is less than $50 \$$. During the testing of the prototype, only the sensor head containing the LD-SSA couple was mounted on a shaker as the shaker could not withstand the load of prototype casing. Note that the shaker has been used to generate the extraneous movements affecting the sensor. 


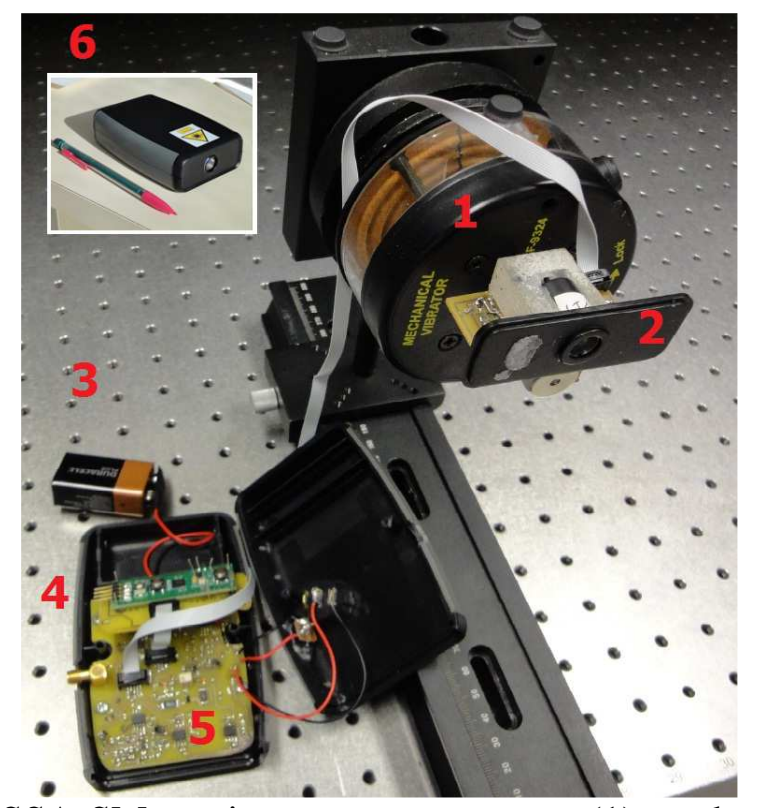

FIGURE 5. Real-time SSA-SM sensing system prototype. (1) mechanical shaker, (2) laser sensor head, (3) battery cell, (4) micro-converter, (5) analog integrators and filters, and (6) Inset picture of prototype.

Let us present some results verifying the operating principle of our real-time robust vibration sensor. A commercial PZT (Piezo-electric Transducer) from Physik Instrumente (P753.2CD) equipped with a built-in capacitive feedback sensor (CFS) of $2 \mathrm{~nm}$ resolution has been used as the target in our set-up (see Fig. 1). The CFS is thus used as a reference sensor.

In Fig. 6, the target has been kept standstill while the parasitic vibration acting on the prototype is at $140 \mathrm{~Hz}$ with a peak-to-peak amplitude of $11.775 \mu \mathrm{m}$. Such a vibration thus results in a corrupted sinusoidal signal indicated in green in Fig. 6 (a). A correct working of the prototype eliminates it as seen by the corrected signal in blue in Fig. 6 (b). Thus, the real-time correction achieved by the data fusion between the two displacement signals is of $31 \mathrm{~dB}$ equivalent to $96 \%$ elimination of the extraneous vibration.

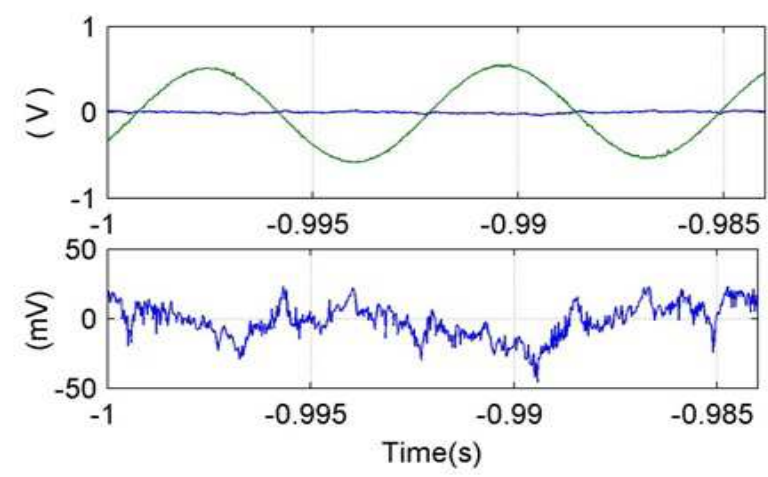

(a)

FIGURE 6. The parasitic vibration is at $140 \mathrm{~Hz}$ with $11.775 \mu \mathrm{m}$ amplitude while the target is standstill. The corrupted (green) and corrected (blue) signals indicate an improvement of $31 \mathrm{~dB}$ or $96 \%$ elimination of the parasitic vibration acting on prototype. 

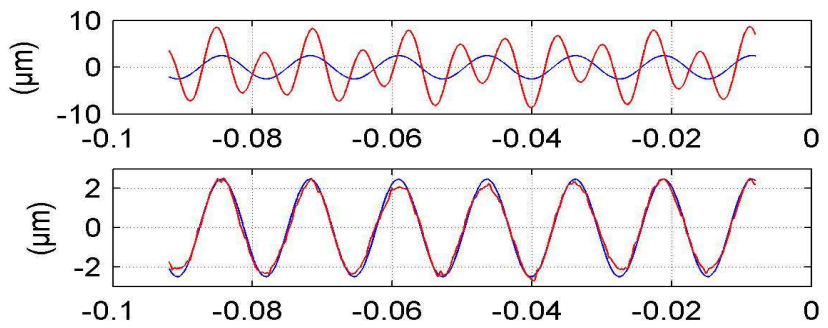

(b)
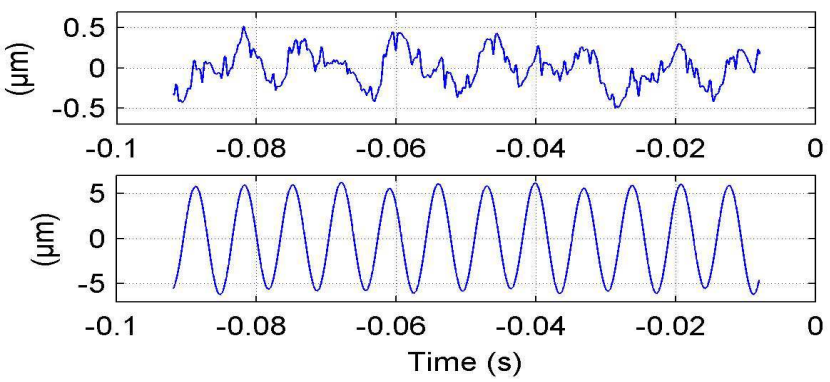

(d)

FIGURE 7. Target vibrates at $79 \mathrm{~Hz}$ with $5 \mu \mathrm{m}$ amplitude while the parasitic vibration is at $140 \mathrm{~Hz}$ with $11.775 \mu \mathrm{m}$ amplitude. (a) corrupted (red) and reference (blue) vibration signals; (b) real-time corrected (red) and reference (blue) signals; (c) error of real-time corrected signal; and (d) error of corrupted signal with respect to the reference signal, respectively.

Now, in Fig. 7, the target vibrates at $79 \mathrm{~Hz}$ with a peak-to-peak amplitude of $5 \mu \mathrm{m}$ while the parasitic vibration remains at $140 \mathrm{~Hz}$ with a peak-to-peak amplitude of $11.775 \mu \mathrm{m}$. The real-time correction achieved by the data fusion between the two displacement signals thus results in recovering a displacement close to that measured by the reference sensor. We thus observe good target vibration recovery with respect to the reference CFS signal in Fig.7 (b). A comparison of the error of real-time corrected signal shown in Fig. 7 (c) with that of the error of corrupted signal shown in Fig. 7 (d) thus indicates a ten-fold improvement achieved by the real-time prototype.

\section{CONCLUSION}

In this paper, a real-time implementation of a Self-Mixing (SM) interferometric laser diode (LD) based vibration sensor coupled with an embedded MEMS (microelectromechanical system) accelerometer has been presented. The said real-time SM vibration sensor system is based on a LD package from Sanyo (DL7140) emitting at $\lambda=785 \mathrm{~nm}$ with an output power of $60 \mathrm{~mW}$. The accelerometer LIS344ALH is from $\mathrm{ST}^{\circledR}$ with a typical noise resolution of $50 \mu \mathrm{g} / \sqrt{\mathrm{Hz}}$. Such a sensor allows measuring correct target movements even when the LD based SM sensor is subject to extraneous movements. This results in a vibration sensing system that can be used for embedded applications as there is no more need of keeping the sensor stationary. Such an approach opens the way for the use of such laser sensors in conditions where the use of antivibration support is not available or possible. The proposed data fusion between the acceleration and SM interferometric signals results in a robust, compact and low-cost sensing system.

\section{ACKNOWLEDGMENTS}

The authors wish to acknowledge the technical support provided by Mr. Clément Tronche and Mr. Francis Jayat for electronic circuit boards and experimental set-up. 


\section{REFERENCES}

1. S. Donati, Electro-optical instrumentation: sensing and measuring with lasers. Upper Saddle River: Prentice Hall PTR, ch. 4, 2004.

2. G. Giuliani, S. Donati, "Laser interferometry" in Unlocking dynamical diversity: optical feedback effects on semiconductor lasers, D. M. Kane and K.A. Shore, Ed. Chichester: John Wiley \& Sons, Ltd, pp. 217-256, 2005.

3. T. Bosch, C. Bes, L. Scalise, and G. Plantier, "Optical feedback interferometry", Encyclopedia of Sensors, American Scientific Publishers, vol. 7, pp. 107-127, 2006.

4. G. Mourat, N. Servagent and T. Bosch, "Distance measurement using the self-mixing effect in a three-electrode distributed Bragg reflector laser diode", Opt. Eng. 39, 738, 2000.

5. A. van der Lee, M. Carpaij, H. Moench, M.Schemmann, and A. Pruijmboom, "A miniaturized VCSEL based sensor platform for velocity measurement", Proc. of the 25th IEEE International Instrumentation and Measurement Technology Conference, pp 141-143, 2008.

6. G. Giuliani, S. Bozzi-Pietra, S. Donati, "Self-mixing laser diode vibrometer", Meas. Sci. Technol., vol. 14, no. 1, pp. 24-32, 2003.

7. Ottonelli, S.; De Lucia, F.; di Vietro, M.; Dabbicco, M.; Scamarcio, G.; Mezzapesa, F.P.; , "A Compact Three Degrees-of-Freedom Motion Sensor Based on the Laser-Self-Mixing Effect," Photonics Technology Letters, IEEE, vol.20, no.16, pp.1360-1362, Aug.15, 2008.

8. Plantier, G.; Bes, C.; Bosch, T.; , "Behavioral Model of a Self-Mixing Laser Diode Sensor," Quantum Electronics, IEEE Journal of , vol.41, no.9, pp. 1157- 1167, Sept. 2005.

9. Bes, C.; Belloeil, V.; Plantier, G.; Gourinat, Y.; Bosch, T.; , "A Self-Mixing Laser Sensor Design With an Extended Kalman Filter for Optimal Online Structural Analysis and Damping Evaluation," Mechatronics, IEEE/ASME Transactions on, vol.12, no.3, pp.387-394, June 2007.

10. C. Bes, G. Plantier, and T. Bosch, "Displacement measurements using a self-mixing laser diode under moderate feedback", IEEE Transactions on Instrumentation and Measurements, vol. 55, no. 4, pp. 1101-1105, 2006.

11. Bosch, T.; El-Assad, J.; Plantier, G.; , "Impact detection in carbon fiber beam using selfmixing sensors," Sensors, 2009 IEEE, vol., no., pp.408-412, 25-28 Oct. 2009.

12. U. Zabit, T. Bosch, and F. Bony "Adaptive transition detection algorithm for a self-mixing displacement sensor", IEEE Sensors Journal, vol. 9, Issue 12, pp. 1879-1886, 2009.

13. U. Zabit, T. Bosch, F. Bony and A. D. Rakic, "A self-mixing displacement sensor with fringe-loss compensation for harmonic vibrations", IEEE Photonics Technology Letters, vol. 22, No. 6, pp. 410-412, 2010.

14. U. Zabit, O. D. Bernal, T. Bosch, and F. Bony, "MEMS accelerometer embedded in a selfmixing displacement sensor for parasitic vibration compensation," Optics Letters, vol. 36, pp. 612-614, 2011.

15. U. Zabit, O. D. Bernal, and T. Bosch, "Self-mixing sensor for real-time measurement of harmonic and arbitrary displacements", Inst. and Meas. Techn. Conf. (I2MTC), 2012 IEEE, 13-16 May 2012, accepted for publication.

16. Zabit, U.; Bernal, O.D.; Bosch, T.; , "A self-mixing displacement sensor compensating parasitic vibration with a MEMs accelerometer," Sensors, 2011 IEEE , vol., no., pp.13861389, 28-31 Oct. 2011. 
17. Norgia, M.; Pesatori, A.; , "Fully analog self-mixing laser vibrometer," Instrumentation and Measurement Technology Conference (I2MTC), 2011 IEEE, vol., no., pp.1-4, 10-12 May 2011. 